\title{
A. Données statistiques disponibles sur Internet
}

\section{Gérard Perroulaz, Tanja Guggenbühl et Xavier Tschumi Canosa}

\section{OpenEdition \\ Journals}

Édition électronique

URL : http://journals.openedition.org/aspd/301

DOI : 10.4000/aspd.301

ISSN : 1663-9669

\section{Éditeur}

Institut de hautes études internationales et du développement

\section{Édition imprimée}

Date de publication : 1 avril 2007

Pagination : 205-215

ISBN : 978-2-88247-066-9

ISSN : 1660-5934

Référence électronique

Gérard Perroulaz, Tanja Guggenbühl et Xavier Tschumi Canosa, «A. Données statistiques disponibles sur Internet », Annuaire suisse de politique de développement [En ligne], 26-1 | 2007, mis en ligne le 09 février 2010, consulté le 08 septembre 2020. URL : http://journals.openedition.org/aspd/301 ; DOI : https://doi.org/10.4000/aspd.301 


\section{A. Données statistiques disponibles sur Internet}

\section{A.1. Flux commerciaux}

\section{A.1.1. Données de l'Administration fédérale des douanes (AFD)}

L'Administration fédérale des douanes (AFD) dispose d'un système de banques de données, duquel est issue la statistique du commerce extérieur de la Suisse. Le site Internet est accessible à l'adresse suivante: <http://www.ezv.admin.ch> $>$ Français $>$ Thèmes $>$ Statistique du commerce extérieur.

\section{$\square$ Disponibilité des données}

Un chapitre de cette page Internet, intitulé Produits, fournit toutes les informations nécessaires pour avoir accès à l'ensemble des données sur le commerce extérieur de la Suisse, en ligne (SWISS-impex) ou sur CD-Rom. Ces produits sont tous payants, mais il est possible de trouver ces données dans certaines bibliothèques suisses via le catalogue RERO ${ }^{1}$. Sinon, le chapitre Commandes permet de commander les CD-Rom. La version papier de cette statistique, qui était également payante, a été abandonnée à partir des données 2006.

Un extrait de toutes les données disponibles sur le commerce extérieur de la Suisse est cependant disponible gratuitement dans les chapitres Vue d'ensemble, Pays et Marchandises de la page Internet précitée. Les chiffres y sont organisés en différents points et peuvent la plupart du temps être visionnés à l'écran et/ou téléchargés en format PDF et/ou Excel.

Les points ci-dessous reprennent ces chapitres plus en détail.

\section{$\square$ Vue d'ensemble}

Ce chapitre fournit traditionnellement les totaux annuels des importations et des exportations ainsi que du solde de la balance commerciale de la Suisse depuis 1980 jusqu'à la dernière année pour laquelle les données sont disponibles. Aucun détail supplémentaire par pays ou par marchandise n'est donné à ce niveau. Un autre tableau de ce chapitre fournit les chiffres du commerce extérieur de la Suisse par habitant, pour les mêmes années.

Dans ce chapitre figurent également les totaux mensuels provisoires des importations et des exportations de l'année complète la plus récente et même jusqu'au dernier mois écoulé. A partir de juin de l'année courante, les chiffres définitifs de l'année précédente sont généralement disponibles.

Dans tous ces chiffres, une différence est faite entre le total 1, sans métaux précieux et pierres gemmes ni objets d'art et antiquités, utilisé principalement pour une observation conjoncturelle, et le total 2, qui reflète l'intégralité du commerce extérieur. 


\section{$\square$ Pays}

Dans ce chapitre, les données sur les importations, les exportations et le solde de la balance commerciale des quatre années les plus récentes sont classées géographiquement, par région économique ou par continent. Les données du commerce extérieur de la Suisse avec ses principaux partenaires sont également disponibles.

Les données par région économique (format Excel) restent agrégées en grands groupes et ne fournissent pas d'information par pays composant ces régions. Pour disposer des données par pays (format Excel), il faut consulter les chiffres par continent.

Les pays qui composent les régions économiques et les continents sont définis dans des documents PDF disponibles dans le chapitre Explications de la page Internet de la statistique du commerce extérieur (rubrique Répertoire des pays).

Wh Mise en garde: la classification des pays en régions économiques retenue par l'Administration fédérale des douanes lui est propre et il convient de l'étudier avec attention avant toute comparaison avec celles issues d'autres sources, en particulier la Liste des bénéficiaires d'APD établie par le CAD (Comité d'aide au développement) de l'OCDE. L'agrégat des pays en développement ou celui des pays en transition qui apparaissent dans les données de l'Administration fédérale des douanes ne sont par exemple aucunement comparables à ceux fournis par la liste du CAD (reproduite à la fin de cet Annuaire).

Référence: dans toute cette partie «Statistiques» de l'Annuaire, c'est la classification de la Liste des bénéficiaires d'APD établie par le CAD qui est utilisée. Pour les flux 2004 ou antérieurs, cette liste était composée de deux parties, la première concernant les pays et territoires en développement et la seconde les pays et territoires en transition. Pour les flux à partir de 2005, la liste ne comporte plus qu'une seule partie, celle des bénéficiaires de l'aide publique au développement.

\section{$\square$ Marchandises}

Dans ce chapitre, les importations et les exportations sont ordonnées selon la nature des marchandises (14 branches principales ${ }^{2}$ de l'économie) ou leur emploi (6 groupes ${ }^{3}$ utilisés pour établir les pronostics de la conjoncture et les comptes nationaux). Les nomenclatures selon la nature ou l'emploi des marchandises sont disponibles dans le chapitre Explications de la page Internet de la statistique du commerce extérieur (rubrique Nomenclatures des marchandises).

Les chiffres concernent les quatre années les plus récentes et sont fournis en format PDF (résumé) ou Excel.

2 Les produits de l'agriculture, de la sylviculture et de la pêche; les produits énergétiques; les textiles, l'habillement et les chaussures; le papier, les ouvrages en papier et les produits des arts graphiques; les cuirs, le caoutchouc et les matières plastiques; les produits des industries chimiques et connexes; les pierres et les terres; les métaux; les machines, les appareils et l'électronique; les véhicules; les instruments de précision, l'horlogerie et la bijouterie; les autres produits divers; les métaux précieux et les pierres gemmes; les objets d'art et les antiquités.

3 Les matières premières et les demi-produits; les produits énergétiques; les biens d'équipement; les biens de consommation; les métaux précieux et les pierres gemmes; les objets d'art et les antiquités. 
Le classement des importations et des exportations selon la nature des marchandises est encore subdivisé par pays, mais seulement les principaux pays de provenance ou de destination pour le groupe de marchandises consulté. Tel n'est pas le cas pour le classement des importations et des exportations selon l'emploi des marchandises, où seule une vue générale est fournie.

Ce point offre encore une vue d'ensemble des importations et des exportations selon la Classification type pour le commerce international (CTCI) ${ }^{4}$. La CTCI, gérée par la Commission de statistique des Nations unies, est une nomenclature de marchandises construite à partir de critères économiques, pour l'établissement des statistiques du commerce extérieur.

\section{$\square$ Analyse du commerce extérieur}

Ce chapitre fournit des informations complémentaires à celles qui sont contenues dans les points précédents. Des textes et des graphiques illustrent l'évolution du commerce extérieur de la Suisse pour l'année des données les plus récentes par rapport à l'année précédente (ou aux années précédentes).

Ce chapitre, dans la rubrique Régions économiques, offre un tableau des 30 principaux pays partenaires de la Suisse pour l'année des données les plus récentes.

\section{$\square$ Autres données}

Les données statistiques sur le commerce extérieur de la Suisse apparaissent également dans d'autres chapitres de la page Internet de l'AFD, comme les importations et les exportations par moyen de transport ou par canton et zone de passage.

Les indices du commerce extérieur pour les années les plus récentes sont présentés dans un de ces chapitres également. Enfin, les trafics spéciaux, qui apparaissent dans un autre chapitre, fournissent notamment les chiffres des exportations suisses de matériel de guerre pour l'année précédant l'année courante (voir également plus bas sous-chap. A.1.3).

\section{A.1.2. Données de la Banque nationale suisse (BNS)}

La Banque nationale suisse (BNS) publie également des données sur le commerce extérieur de la Suisse, dans son Bulletin mensuel de statistiques économiques : <http://www.snb.ch> > Français >Publications >Bulletin mensuel de statistiques économiques.

Le chapitre I (lettre $I$ ) fournit les chiffres sur le commerce extérieur, selon l'utilisation des marchandises, selon la nature des marchandises et par pays. Les données sont disponibles en format PDF (mises en forme telles qu'elles apparaissent dans la publication), ou en format de données (Excel ou Text). La publication fournit les totaux annuels des importations, des exportations et du solde de la balance commerciale.

Limite: en format de données, les chiffres mensuels doivent être additionnés manuellement pour obtenir les totaux par année.

4 Voir $<$ http://unstats.un.org > > Statistical Databases $>$ Classifications Registry >Quicklinks $>$ SITC Rev.4 (en anglais). 
A la différence des chiffres de l'Administration fédérale des douanes, qui fournit les données sur le commerce extérieur d'année en année (à la mi-année pour l'année antérieure), la BNS fournit les données de mois en mois (avec un délai de trois mois - au mois de février pour les données du mois de novembre précédent p. ex.). Les chiffres de la BNS correspondent au total 1 retenu par l'Administration fédérale des douanes, soit ceux n'incluant pas les métaux précieux et pierres gemmes ni les objets d'art et antiquités.

Les chiffres de la BNS n'offrent aucun détail par pays sur le commerce extérieur de la Suisse avec les pays en développement, sauf pour l'Inde.

Wh Mise en garde: la liste des pays en développement de la BNS n'est pas la 2 même que celle qui est retenue dans les chapitres B et C de la présente partie «Statistiques» de l'Annuaire et qui est fondée sur la Liste des bénéficiaires d'APD établie par le CAD (Comité d'aide au développement) de l'OCDE (liste reproduite à la fin de cet Annuaire).

\section{A.1.3. Données du Secrétariat d'Etat à l'économie (seco)}

Le Secrétariat d'Etat à l'économie (seco) offre sur son site Internet des informations par pays concernant les relations économiques extérieures de la Suisse: <http://www.seco.admin.ch> > Français > Thèmes > Politique économique extérieure >Information par pays. Ces informations par pays sont des fiches en format Word régulièrement mises à jour, contenant des données sur le pays en question et dépassant le strict cadre du commerce extérieur de la Suisse (p. ex. données géopolitiques, liens diplomatiques entre la Suisse et le pays).

Un document intitulé Liste de sources «Relations économiques bilatérales » est accessible en téléchargement sur cette page Internet du seco. Il fournit des informations complémentaires aux fiches, sous la forme de liens Internet accessibles gratuitement et pointant vers des données économiques par pays.

Une page du site Internet du seco fournit également des informations sur les contrôles à l'exportation, notamment en ce qui concerne le matériel de guerre: <http://www.seco.admin.ch> > (Français) > Thèmes >Contrôles à l'exportation >Matériel de guerre. Cette page présente, dans sa partie News, les chiffres des exportations de matériel de guerre par pays de destination, dès le mois de février pour l'année d'avant. Les chiffres des années antérieures peuvent être trouvés en suivant le lien Statistiques précédentes.

\section{A.2. Flux financiers}

\section{A.2.1. Données de la Banque nationale suisse (BNS)}

Le site Internet de la Banque nationale suisse (BNS) publie les informations les plus complètes sur les flux financiers en provenance ou à destination de la Suisse: <http://www.snb.ch>>Français >Données et statistiques. Ce site permet d'accéder aux publications de la BNS, en fonction de mots clés ${ }^{5}$. Chacun

5 Statistiques bancaires, agrégats monétaires, données sur les marchés des changes, de l'argent et des capitaux, balance des paiements, position extérieure nette, dette extérieure, investissements directs, données macroéconomiques. 
d'eux est accompagné d'un court descriptif du contenu des publications pertinentes, ce qui fait de cette page Internet un outil de recherche très utile.

La liste des publications à proprement parler se trouve à la page suivante du site Internet de la BNS : <http://www.snb.ch>> Français >Publications.

\section{$\square$ «Bulletin mensuel de statistiques économiques »}

En activant le lien <http://www.snb.ch> > Français > Publications > Bulletin mensuel de statistiques économiques, c'est le Bulletin mensuel de statistiques économiques le plus récent qui est présenté mais tout en bas de la page un autre lien permet d'accéder aux bulletins mensuels archivés depuis l'année 2000. Tous les chiffres sont disponibles en format PDF mais également en format de données (Excel ou Text).

Le chapitre F du bulletin, consacré au marché des capitaux, offre les chiffres concernant les emprunts de débiteurs étrangers en Suisse, en souscription publique ou non, par catégorie d'emprunt ou par groupe de pays.

Le chapitre $\mathrm{S}$ du bulletin, consacré aux investissements directs, offre les chiffres concernant les investissements directs suisses à l'étranger. Les flux annuels de ces investissements (exportations de capitaux), les stocks d'investissements (état en fin d'année) ainsi que l'effectif du personnel dans les entreprises suisses à l'étranger sont disponibles par pays ou par branche/secteur d'activité.

MM Mise en garde: la classification des pays utilisée par la BNS lui est propre 4 et ne doit pas être comparée avec celles d'autres sources, en particulier la Liste des bénéficiaires d'APD établie par le CAD (Comité d'aide au développement) de l'OCDE. Certains pays classés par la BNS dans le groupe des pays nouvellement industrialisés sont par exemple considérés comme des pays en développement par le CAD.

Référence: dans toute la partie «Statistique» de l'Annuaire, c'est la classification de la liste des bénéficiaires de l'aide établie par le CAD qui est utilisée. Pour les flux 2004 ou antérieurs, cette liste était composée de deux parties, la première concernant les pays et territoires en développement et la seconde les pays et territoires en transition. Pour les flux à partir de 2005, la liste ne comporte plus qu'une seule partie, celle des bénéficiaires de l'aide publique au développement.

\section{$\square$ «L'évolution des investissements directs»}

Ce rapport sur les investissements directs paraît annuellement en annexe au numéro de décembre du Bulletin mensuel de statistiques économiques (voir cidessus) : <http://www.snb.ch > > Français > Données et statistiques >Investissements directs $>$ L'évolution des investissements directs. En activant ce lien du site Internet de la BNS, la publication est disponible depuis l'année 2000 jusqu'à l'année la plus récente (en décembre 2006 a été publiée L'évolution des investissements directs en 2005). Cette publication fournit des tableaux et des commentaires sur l'évolution des investissements directs suisses à l'étranger et des investissements directs étrangers en Suisse pour l'année de référence. 


\section{$\square$ «Les banques suisses»}

Cette publication, qui paraît annuellement en été, est disponible en activant le lien correspondant dans la rubrique des publications du site Internet de la BNS : <http://www.snb.ch > Français > Publications > Les banques suisses. Les éditions sont disponibles depuis 1996 en format PDF (publication complète ou résumé) et seule la publication la plus récente propose en plus des chiffres en format Excel ou Text.

Le chapitre 1.5 de cette publication fournit la répartition par pays des avoirs et engagements à l'étranger figurant dans les bilans d'une centaine de banques suisses (tableau 32).

Définition: les opérations figurant au bilan d'une banque sont seulement celles que la banque effectue pour son propre compte.

Le chapitre 2.2 de cette publication fournit la répartition par pays des avoirs et des engagements à l'étranger de cette même centaine de banques suisses, mais à titre fiduciaire (tableau 38).

Définition: les opérations à titre fiduciaire d'une banque sont celles que la banque effectue pour le compte et aux risques de ses clients.

Wh Mise en garde: ce chapitre ne couvre pas l'ensemble des avoirs et engagements à l'étranger, car toutes les banques n'y figurent pas et le secteur parabancaire (p. ex. gérants de fortune, agents fiduciaires, bureaux de change) n'y est pas inclus. De plus, les tableaux 32 et 38 de cette publication sont les seuls qui fournissent des chiffres par pays.

\section{$\square$ Balance suisse des paiements et position extérieure nette de la Suisse}

Trois annexes du Bulletin mensuel de statistiques économiques apparaissent dans la rubrique suivante des publications de la BNS: <http://www.snb.ch> $>$ Français >Publications > Balance des paiements et position extérieure nette de la Suisse. Il s'agit de L'évolution des investissements directs, présentée plus haut, ainsi que de

• l'annexe Balance suisse des paiements, qui paraît une fois par an ${ }^{6}$ et qui est disponible sur le site Internet de la BNS en format PDF depuis 1996. Elle commente l'évolution des flux économiques et financiers entre la Suisse et l'étranger de l'année sous revue (flux de biens et services, flux de capitaux) et est assortie de tableaux fournissant les chiffres avec une profondeur historique de cinq ans ;

- l'annexe Position extérieure nette de la Suisse, disponible en format PDF pour chaque année ${ }^{7}$ depuis 2000 . Elle commente l'évolution des chiffres des actifs à l'étranger, des passifs envers l'étranger et de la position nette de la Suisse, selon les directives du Fonds monétaire international (FMI). Les tableaux fournissent également ces chiffres avec une profondeur historique de cinq ans.

Parution fin septembre d'une année sur les chiffres de l'année antérieure.

Parution fin décembre d'une année sur les chiffres de l'année antérieure. 


\section{A.2.2. Données du Comité d'aide au développement (CAD) de l'OCDE}

Le Comité d'aide au développement (CAD) de l'OCDE possède un site Internet en anglais riche en données statistiques: <http://www.oecd.org/dac> > Aid Statistics. Ce site est beaucoup plus complet que celui en langue française (<http://www.oecd.org/cad> > Statistiques de l'aide) et, lorsqu'un document existe dans l'autre langue, une référence le mentionne et un lien conduit à la traduction.

\section{$\square$ Disponibilité des données}

Le CAD met ses données à disposition sous forme de tableaux accessibles gratuitement en ligne, mais également sous forme de CD-Rom (en particulier le CD-Rom des Statistiques sur le développement international - International Development Statistics CD-ROM) ou de rapports papier qui peuvent être achetés dans la librairie en ligne de l'OCDE ${ }^{8}$ ou consultés dans les bibliothèques suisses via le catalogue RERO9.

Coopération pour le développement. Rapport 2006, Les Dossiers du CAD, Paris, OCDE, 2006.

\section{$\square$ Annexe statistique du rapport annuel «Coopération pour le développement»}

L'annexe statistique du rapport Coopération pour le développement est disponible en ligne, en français ou en anglais (statistical Annex of the Development Co-operation Report), et offre, en une quarantaine de tableaux en format Excel, une vue complète sur les flux financiers entre les pays membres du CAD et les pays bénéficiaires de l'aide.

La table des matières (Table of Contents) de cette annexe est utile pour trouver les tableaux recherchés.

MM Mise en garde: les tableaux de l'annexe statistique du rapport annuel Coopération pour le développement ne sont pas toujours numérotés de la même manière d'une année à l'autre et c'est la raison pour laquelle la table des matières fait foi.

Le tableau 13 de l'annexe du rapport 2006 présente les flux financiers entre les pays membres du CAD et les pays bénéficiaires de l'aide pour l'année 2005 en quatre catégories:

- l'aide publique au développement (APD - voir sous-chap. A.3.1 ci-dessous);

$\checkmark$ les autres apports du secteur public (accordés aux conditions du marché, contrairement à l'APD);

$\checkmark$ les dons des organismes privés bénévoles;

- les capitaux privés qui sont versés à ces pays aux conditions du marché (investissements directs, crédits à l'exportation et investissements de portefeuille).

Le tableau 14 fournit les mêmes données, mais pour les années 2002 à 2005 ainsi que pour la moyenne des années 1994-1995, soit dix ans avant les dernières données disponibles. 
Le tableau 37 présente les taux de change moyens annuels (de 2001 à 2005 dans le rapport 2006) vis-à-vis du dollar pour les devises des pays membres du CAD, utiles lors de comparaisons avec les chiffres d'autres sources non libellés en dollars.

Le tableau 38 fournit les chiffres du revenu national brut (RNB) et de la population pour chaque pays du CAD, permettant ainsi de calculer ce que représentent les différentes catégories de flux par unité de RNB ou par habitant (dans le rapport 2006 : de 2003 à 2005 ainsi que la moyenne des années 1994 et 1995).

\section{$\square$ Statistiques sur le développement international en ligne}

Le site Internet des données statistiques du CAD contient un lien vers la page des statistiques sur le développement international en ligne (International Development Statistics Online), qui offre un accès aux banques de données du CAD (Datasets) sur les flux financiers à destination des pays bénéficiaires de l'aide.

Depuis mai 2006, un nouveau système, intitulé OECD.Stat (prononcer «OECD dot Stat»), agrège ces statistiques et offre aux utilisateurs un accès facilité aux catégories de flux financiers vers les pays bénéficiaires de l'aide présentées cidessus. Les données peuvent être filtrées par pays donneur et exportées en format Excel. Il est aussi possible d'obtenir de ce système les données sur une très longue période, soit depuis 1960.

\section{$\square$ Autres données statistiques du CAD ou de l'OCDE}

Le CAD propose également sur la page des données statistiques de son site Internet des fiches synthétiques sur les différents flux ou montants pertinents, pour les pays membres du CAD (aide par donneur - donor aid charts) et pour les pays bénéficiaires de l'aide (aide par bénéficiaire - recipient aid charts).

La Liste des bénéficiaires d'APD établie par le CAD (DAC List of ODA Recipients) est également disponible sur cette page Internet.

MM Mise en garde: cette liste est mise à jour régulièrement et à chaque année de flux financiers (notamment ceux d'APD) correspond une liste précise, valable pour l'année en question. Pour les flux 2004 ou antérieurs, la liste était composée de deux parties, la première concernant les pays et territoires en développement et la seconde les pays et territoires en transition. Pour les flux à partir de 2005, la liste ne comporte plus qu'une seule partie, celle des bénéficiaires de l'aide publique au développement.

\section{A.2.3. Composantes des flux financiers à long terme destinés aux pays en développement}

Chaque année, dans la partie Sources and Definitions de sa publication Global Development Finance ${ }^{10}$, la Banque mondiale offre un schéma et un tableau qui permettent de mieux comprendre la composition des flux financiers intéressant les pays en développement. Le schéma présente les composantes des flux financiers à long terme destinés aux pays en développement et le tableau situe ces

10 Edition la plus récente: World Bank, Global Development Finance 2006 : The Development Potential of Surging Capital Flows, II. Summary and Country Tables, Washington, 2006, pp. xxv-xxvi. 
flux financiers dans l'ensemble des flux financiers à long terme qui entrent dans les différentes balances intermédiaires de la comptabilité nationale (balance des transactions courantes, balance des capitaux et balance financière) et qui s'équilibrent dans la balance des paiements.

Annuaire 2006, nº 1, schéma 2 et tableau 1, p. 219.

\section{A.3. Flux d'aide au développement}

\section{A.3.1. Données du Comité d'aide au développement (CAD) de l'OCDE}

Comme décrit dans le sous-chapitre A.2.2 ci-dessus, le Comité d'aide au développement $(\mathrm{CAD})$ de l'OCDE possède un site Internet riche en données statistiques, <http://www.oecd.org/dac> >Aid Statistics. Le même sous-chapitre informe sur la disponibilité des données du CAD.

\section{$\square$ Annexe statistique du rapport annuel «Coopération pour le développement»}

L'annexe statistique du rapport Coopération pour le développement est disponible en ligne, en français ou en anglais (statistical Annex of the Development Co-operation Report), et offre, en une quarantaine de tableaux en format Excel, une vue complète sur les flux financiers entre les pays membres du CAD et les pays bénéficiaires de l'aide.

La table des matières (Table of Contents) de cette annexe est utile pour trouver les tableaux recherchés.

Mise en garde: les tableaux de l'annexe statistique du rapport annuel Coopération pour le développement ne sont pas toujours numérotés de la même manière d'une année à l'autre et c'est la raison pour laquelle la table des matières fait foi.

Le graphique 1 de l'annexe du rapport 2006 présente une vue d'ensemble des contributions d'aide publique au développement (APD) consenties par les pays membres du CAD en 2005, en valeur absolue et en pourcentage du RNB. Le tableau 1 fournit les chiffres de ces contributions pour les années 2004 et 2005.

Définition: le CAD donne une définition de l'agrégat d'aide publique au développement (APD) qui peut être trouvée dans les notes techniques à la fin des rapports annuels sur la coopération pour le développement (disponibles en version papier à l'achat ou en bibliothèque, mais pas directement en ligne). Cette définition peut être résumée ainsi : l'APD désigne les prêts et dons accordés à des conditions financières libérales par le secteur public des pays membres du CAD aux pays et territoires figurant dans la Liste des bénéficiaires d'APD établie par le $C A D$, dans le but principal de faciliter le développement économique et d'améliorer les conditions de vie dans ces pays.

WM Mise en garde: les données sur l'agrégat d'aide publique (AP), qui était destinée aux pays et territoires en transition, n'existent plus dans l'annexe statistique pour les flux à partir de l'année 2005.

Le tableau 14 de l'annexe du rapport 2006 fournit les détails des flux composant l'agrégat d'APD pour chaque pays membre du CAD et pour les quatre années de données les plus récentes, en particulier les flux d'APD bilatérale (prêts et 
dons) et ceux d'APD multilatérale. Ce tableau indique également les flux privés des organismes privés bénévoles vers les pays en développement.

Pour avoir une répartition des flux par pays bénéficiaire de l'aide en provenance de l'ensemble des pays membres du CAD sur les cinq années les plus récentes, il faut se reporter au tableau 25 (APD en faveur des pays en développement).

Le tableau 32 présente la liste des 15 principaux pays bénéficiaires de l'APD, pour chaque pays membre du CAD, en pourcentage du total de l'APD versée (versements bruts) en 1984-85, 1994-95 et 2004-05. Il permet de comparer la focalisation géographique de l'aide propre à chaque pays donneur dans le temps et entre les pays membres du CAD. La répartition de l'APD bilatérale entre les catégories de pays retenues dans la Liste des bénéficiaires d'APD établie par le $C A D$ et par entité géographique apparaît également dans le tableau 32, pour chaque pays membre du CAD.

Définitions : un versement brut correspond au montant total versé au cours d'une période comptable donnée. Un versement net correspond au versement brut déduction faite de tous les remboursements de principal ou des restitutions de dons intervenus pendant la même période comptable.

\section{A.3.2. Données de la Direction du développement et de la coopération (DDC)}

La Direction du développement et de la coopération (DDC) publie chaque année des données statistiques sur l'aide de la Suisse aux pays en développement. Ces données sont publiées séquentiellement et les chiffres ne sont pas forcément les mêmes entre les différentes sources (actualisation de chiffres provisoires).

\section{«Rapport annuel de la coopération internationale de la Suisse»}

Le rapport annuel est publié conjointement par la DDC et le Secrétariat d'Etat à l'économie (seco), qui sont les deux services fédéraux chargés de coordonner et de mettre en œuvre la coopération internationale de la Suisse. Il paraît au milieu de l'année qui suit l'année de référence sur laquelle portent les statistiques (en juillet 2006 est paru le rapport 2005, avec des chiffres provisoires pour 2005 et des chiffres actualisés pour 2004).

MM Mise en garde: s'agissant d'une actualisation des chiffres 2004, ceux-ci ne sont pas forcément les mêmes que ceux qui avaient été publiés dans le rapport 2004 une année auparavant. Les chiffres 2005 étant provisoires, ils ne sont pas non plus forcément les mêmes que les chiffres actualisés qui sont publiés plusieurs mois plus tard dans l'ouvrage Aide de la Suisse aux pays en développement (voir plus bas).

Le rapport est disponible gratuitement en version électronique (PDF) et peut être commandé en version papier sur le site Internet de la DDC: <http://www. ddc.admin.ch $>>$ Documentation.

La première partie, thématique, de ce rapport passe en revue les activités de la Suisse en matière de coopération internationale en 2005. La seconde partie, statistique, fournit les chiffres de l'APD de la Suisse pour l'année de référence du rapport et pour l'année précédente. Cette partie statistique du rapport fournit 
entre autres aussi les chiffres des dépenses d'aide bilatérale de la DDC et du seco par pays, ceux de la DDC pour l'aide humanitaire et par secteur d'activité ainsi que ceux du seco par instrument.

\section{«Aide de la Suisse aux pays en développement»}

Cette publication de la DDC, en version bilingue français et allemand, est disponible généralement au cours du printemps et porte sur les chiffres de deux ans auparavant (au printemps 2007 paraît l'ouvrage de statistiques 2005). Ces données ne sont disponibles pour le moment qu'en version papier, mais un exemplaire de l'ouvrage peut être obtenu gratuitement auprès de la DDC ${ }^{11}$. Cette publication, intitulée Aide de la Suisse aux pays en développement et en transition jusqu'à l'édition parue en 2006, paraît en 2007 sous le titre Aide de la Suisse aux pays en développement. Statistiques 2005, mais elle inclut tout de même les flux d'aide aux pays du Sud et de l'Est non compris dans la Liste des bénéficiaires d'APD établie par le CAD.

Mh Mise en garde: cet ouvrage étant publié plusieurs mois après le Rapport annuel de la coopération internationale de la Suisse (voir plus haut), les chiffres fournis sont actualisés et ne sont plus provisoires. Ils peuvent donc être différents pour l'année commune de référence.

Les flux d'aide suivants sont présentés dans ce document:

๖ aide publique et privée aux pays en développement: vue d'ensemble, évolution, selon la source de financement;

๑ contributions multilatérales entrant dans l'APD;

$\square$ flux financiers bilatéraux vers les pays bénéficiaires de l'APD et les autres pays du Sud et de l'Est: par continent, par catégorie de pays selon la classification du CAD et par pays. Ce tableau est une version abrégée de celui se trouvant dans le chapitre B de la présente partie «Statistique»;

$\checkmark$ contributions bilatérales: aide publique et privée bilatérale par continent et par pays ;

$\square$ aide publique des cantons aux pays bénéficiaires de l'APD et aux autres pays du Sud et de l'Est: par canton;

\ aide publique des communes aux pays bénéficiaires de l'APD et aux autres pays du Sud et de l'Est: par canton et par commune;

๖ aide privée des organismes bénévoles (ONG) aux pays bénéficiaires de l'APD et aux autres pays du Sud et de l'Est: par organisme et par pays bénéficiaire de l'aide.

A la fin de ce document se trouvent encore la Liste des bénéficiaires d'APD établie par le CAD ainsi qu'un répertoire des organismes privés bénévoles (ONG) œuvrant dans le domaine de l'aide (avec leurs coordonnées de contact). 